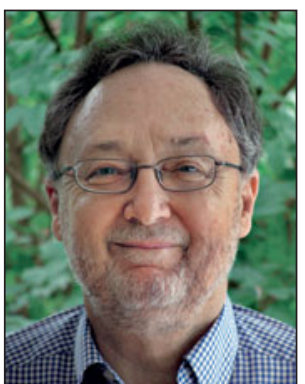

David Larbalestier

Guest Editor for this issue of MRS Bulletin

Applied Superconductivity Center, National High Magnetic Field Laboratory, Department of Mechanical Engineering, Florida State University, 2031 E. Paul Dirac Drive, Tallahassee, FL 32310, USA; tel. 850-645-7483; and email larbalestier@asc.magnet.fsu.edu. Larbalestier is the Francis Eppes Professor of Superconducting Materials in the Department of Mechanical Engineering, director of the Applied Superconductivity Center, and chief materials scientist in the National High Magnetic Field Laboratory at Florida State University. He has worked in superconductivity for the majority of his professional career, being especially interested in the development of conductors and prototype magnets of new superconductors. He has been deeply involved in the practical development of $\mathrm{Nb}-\mathrm{Ti}_{\mathrm{i}} \mathrm{Nb}_{3} \mathrm{Sn}$, $\mathrm{Bi}-2223, \mathrm{YBCO}, \mathrm{MgB}_{2}$, and most recently Bi-2212 conductor forms. Larbalestier is a fellow of the American Physical Society, the Institute of Physics, a member of the National Academy of Engineering, and an author of more than 350 publications on superconductivity with his students, postdoctoral researchers, and scientific colleagues.

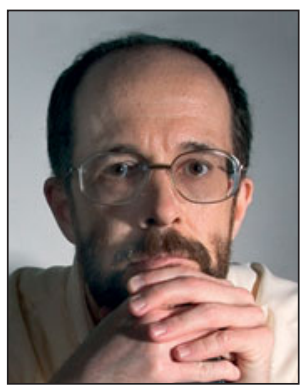

\section{Paul C. Canfield}

Guest Editor for this issue of MRS Bulletin

59 Physics, Department of Physics, lowa State University, Ames, IA 50011, USA; tel. 515294-6270; and email canfield@ameslab.gov. Canfield is a senior physicist at the U.S. Department of Energy's Ames Laboratory and a distinguished professor of physics at lowa State University. He received his $\mathrm{PhD}$ degree from UCLA (1990) and performed postdoctora research at Los Alamos National Laboratory. His research broadly focuses on the electronic and magnetic states of crystalline, intermetallic systems. He has worked extensively on superconductors, heavy fermions, local moment ordering, spin glasses, quasicrystals, and metal-to-insulator transitions. He is a fellow of the American Physical Society and currently holds the Robert Allen Wright Endowed Professorship in Physics.

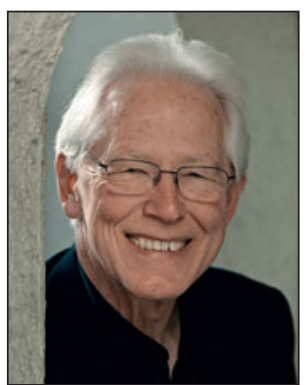

\section{Malcolm R. Beasley}

Geballe Laboratory for Advanced

Materials, Stanford University, Stanford,

CA 94305, USA; tel. 650-723-1196;

and email beasley@stanford.edu.

Beasley is the Sidney and Theodore Rosenberg Professor of Applied Physics and has served as dean of the School of Humanities and Sciences at Stanford University. He received both his bachelor's degree in engineering physics and his $\mathrm{PhD}$ degree in physics from Cornell University. He then joined the faculty in the Division of Engineering and Applied Physics at Harvard University, achieving the rank of associate professor before moving to Stanford. Beasley's research has primarily focused on superconductivity and is currently aimed at the search for new and better higher temperature superconductors. He is a member of the National Academy of Sciences and became an emeritus professor in 2010.

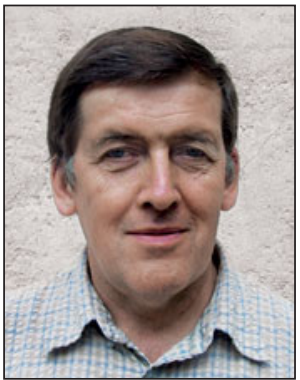

Sergey L. Bud'ko

A111 Physics Hall, lowa State University,

Ames, IA 50011, USA; tel. 515-294-3986;

and email budko@ameslab.gov.

Bud'ko is a scientist at the Ames Laboratory, United States Department of Energy, and an adjunct associate research professor at lowa State University. He received his PhD degree in experimental solid-state physics from the Moscow Institute of Physics and Technology (1986). His research interests include thermodynamic and transport properties of novel materials and materials with novel ground states in multi-extreme conditions (high pressure, high magnetic fields, low temperature); superconductivity, quantum oscillations, density waves, metamagnetism, strong electronic correlations, and quantum criticality. Bud'ko is a fellow of the American Physical Society.

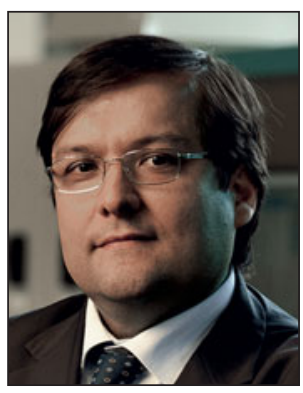

\section{Giovanni Grasso}

Columbus Superconductors SpA,

Via delle Terre Rosse 30, 16133

Genova, Italy; tel. 39-010-8698100;

and email grasso.gianni@clbs.it.

Grasso is general director at Columbus Superconductors in Genova, Italy. He graduated with a degree in physics from the University of Genova in 1992 and received his PhD degree from the University of Geneva, Switzerland, in 1997. He has been active for nearly two decades in the development and characterization of hightemperature superconducting materials for applications. Grasso also was co-founder of Columbus Superconductors in 2003 and has been general director there since 2006 .

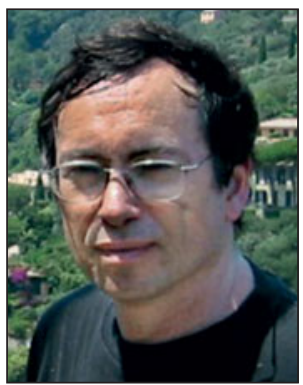

\section{Alex Gurevich}

Department of Physics, Old Dominion University, Norfolk, VA 23529, USA; tel. 757683-4394; and email gurevich@odu.edu. Gurevich is a professor in the Department of Physics of Old Dominion University. He obtained his $\mathrm{PhD}$ degree in physics from the USSR Academy of Science in 1983. He was a staff scientist at the Institute of High Temperatures in Moscow, Kernforschungszentrum Karlsruhe, the University of Wisconsin-Madison, and the National High Magnetic Field Laboratory. His research is in theoretical condensed matter physics and superconductivity. Gurevich is a co-author of two books and more than 140 publications. He is also a fellow of the American Physical Society.

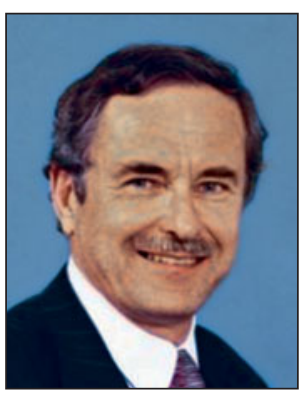

\section{Alexis P. Malozemoff}

American Superconductor Corp., 64 Jackson Rd., Devens, MA 01434-4020, USA; tel. 978 842-3331; and email amalozemoff@ amsc.com.

Malozemoff is currently technical advisor at American Superconductor (AMSC). With a BA degree in chemistry and physics from Harvard University and a PhD degree in materials science and engineering from Stanford University, he is an expert in magnetism and superconductivity, with 200 technical publications, and co-discoverer of "giant flux creep" and the irreversibility line in high-temperature superconductors. From 1971 to 1990, Malozemoff was an IBM Research staff scientist, manager, and senior manager. From 1991 to 2009, he was AMSC's vice president for research and development and its chief technical officer-leading conductor, applications, and patent portfolio development. He is also a fellow of the American Physical Society and IEEE. 


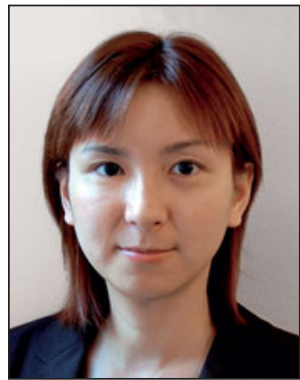

$\mathrm{Ni} \mathrm{Ni}$

Department of Chemistry, Princeton University, Princeton, NJ 08544, USA; tel. 609-2585556; and email nni@princeton.edu.

$\mathrm{Ni}$ is a postdoctoral research associate in the Department of Chemistry at Princeton University. She received her $\mathrm{PhD}$ degree with a major in condensed matter physics and a minor in materials science and engineering from lowa State University in 2009. Her research is focused on the discovery, synthesis, and characterization of novel superconductors and magnetic materials. Ni has published around 80 peer reviewed papers with more than 2,000 citations. She was awarded the research excellence award from lowa State University in 2009 and the inventor incentive award from the Ames Laboratory, U.S. Department of Energy in 2011.

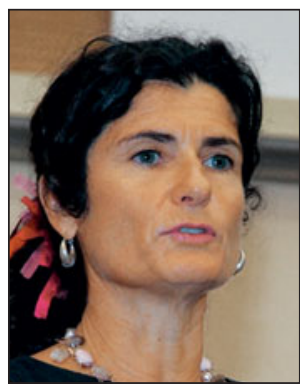

Marina Putti

CNR-SPIN and Dipartimento di Fisica,

Università di Genova via Dodecaneso

33, 16146 Genova, Italy; tel. 39-010-

3536383; and email putti@fisica.unige.it.

Putti is an associate professor at the University of Genova, Italy, and leader of the Superconductivity Team of the CNR-SPIN Institute. She graduated from the University of Firenze in 1986 with a degree in physics and received her $\mathrm{PhD}$ degree from the University of Genova in 1990. Putti's scientific interests cover various fields such as thermal, electrical, and magnetic properties of superconductors. Her main scientific results were obtained on the effects of doping and irradiation in $\mathrm{MgB}_{2}$ and Fe-based superconductors. She also has played a leading role at national and international levels in the investigation of $\mathrm{MgB}_{2}$ and more recently of Fe-based superconductors.

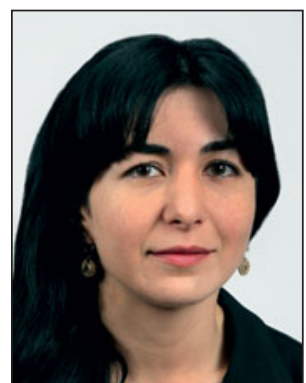

\section{Athena Safa-Sefat}

Oak Ridge National Laboratory, 1 Bethel

Valley Rd., PO Box 2008, Oak Ridge,

TN 37831-6056, USA; tel. 865-574-

5495; and email sefata@ornl.gov.

Sefat is a research staff scientist at Oak Ridge National Laboratory (ORNL). She received her PhD degree from McMaster University in 2005. Her doctorate thesis focused on the magnetic and electronic transitions of vacancy-doped oxide crystals. Her education was broadened at Ames Laboratory, where she spent two years as a postdoctoral fellow searching for new magnetic intermetallic materials. She joined ORNL as a Wigner Fellow in December 2007 and is currently a staff member in the Materials Science and Technology Division. Her scientific interests lie in the study of promising materials for energy-related applications, such as those of high-temperature superconductors. In her laboratory, preparation of promising materials is crucial. Subsequent to this, a variety of physical and magnetic property techniques is used to evaluate the materials' thermodynamic and transport behaviors. In January 2010, Sefat received an Early Career Research Program fund from the U.S. Department of Energy to study promising superconductors.

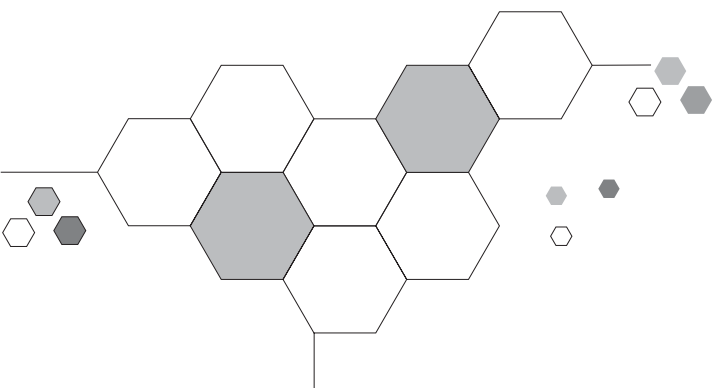

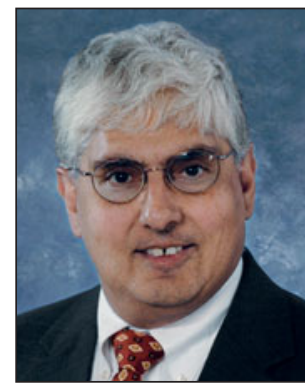

David Joseph Singh

Oak Ridge National Laboratory, MS-6114 Oak Ridge, TN 37831-6114, USA; tel. 865-

241-1944; and email singhdj@ornl.gov.

Singh is a corporate fellow at Oak Ridge National Laboratory (ORNL). He has a PhD degree in physics from the University of Ottawa and did postdoctoral work at the College of William and Mary and the Naval Research Laboratory. Before joining ORNL, he was a staff member and section head at the Naval Research Laboratory. Singh also is a fellow of the American Physical Society and a recipient of the E.O. Hulburt Award.

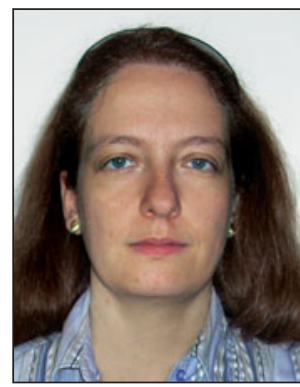

Chiara Tarantini

National High Magnetic Field Laboratory, 2031 E. Paul Dirac Drive, Tallahassee, FL 32310, USA; tel. 850-645-7491; and email tarantini@asc.magnet.fsu.edu.

Tarantini is an assistant scholar scientist in the Applied Superconductivity Center at the National High Magnetic Field Laboratory. She received her $\mathrm{PhD}$ degree in physics from the University of Genoa, Italy, in 2007 and subsequently worked at the CNR-INFM (Italian National Institute of Matter Physics). Her primary research is in the field of superconductivity studying both fundamental aspects of new materials and properties for applications.

\section{Cryogenic Systems}

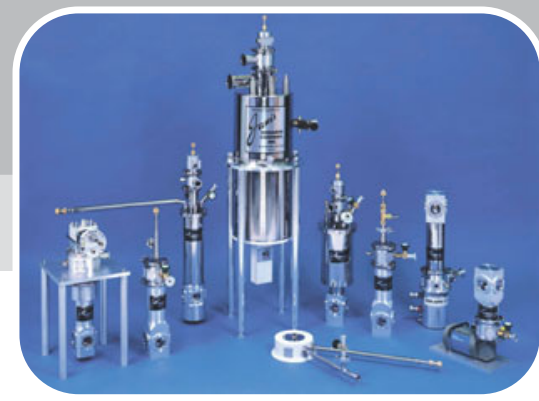

Does your research require low temperatures? Contact Janis today. Our engineers will assist you in choosing the best system for your application.

-10
$-\mathrm{C}$

$10 \mathrm{mK}$ to $800 \mathrm{~K}$

Cryocoolers

- Magnet Systems

LHe/LN 2 Cryostats

Dilution Refrigerator Systems

Micro-manipulated Probe Stations 


\section{$\operatorname{Titan}^{3^{\mathrm{TM}}}$ G2 60-300 \\ Ultimate performance and flexibility}

Plasmon imaging in STEM/EELS

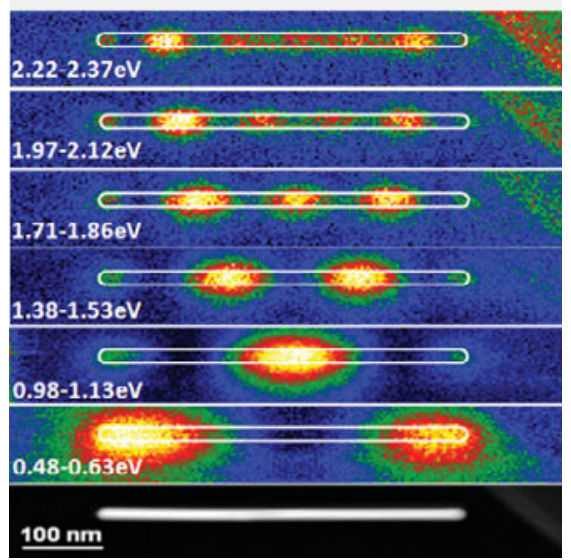

STEM/EELS map of low energy loss region of Ag nano-antenna using a monochromator. The relationship between the spatial and energy distribution is measured down to $0.55 \mathrm{eV}$.

Courtesy of D. Rossouw, M. Couillard, J. Vickery, E. Kumacheva, G.A. Botton. Also in NanoLetters, 29 March 2011, dx.doi.org/10.1021/n1200634w
Atomic imaging of light elements

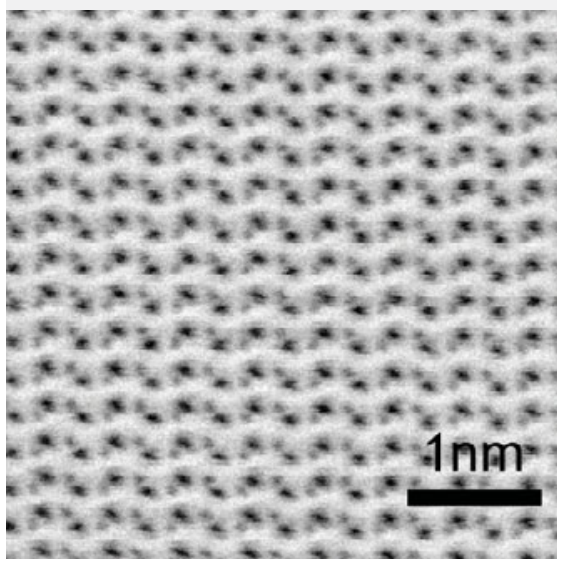

Angular brightfield STEM imaging (ABF) on $\mathrm{GaN}$ in [11-20] projection. The $\mathrm{Ga}$ and $\mathrm{N}$ dumbbell distance can be resolved atomically (raw data).
Atomic chemical mapping by EDX

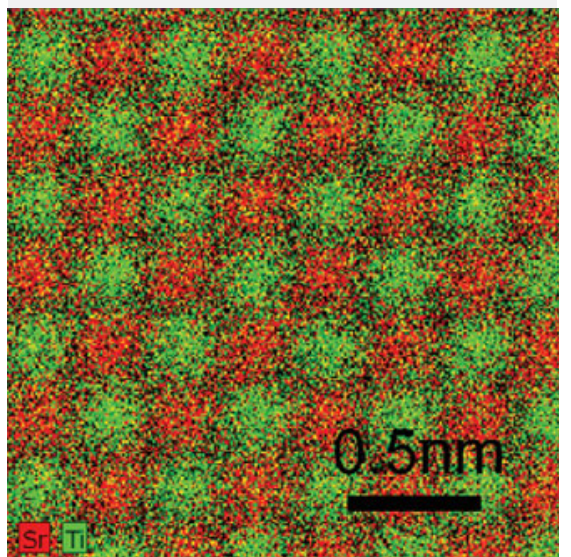

Composite strontium and Titanium image extracted from the Sr-L and Ti-K EDS signal $256 \times 256$ pixels, 10 ms dwell time/pixel (raw data).

Sample courtesy of C. Jia, The Ernst-Ruska

Centre for Microscopy and Spectroscopy with Electrons, Germany

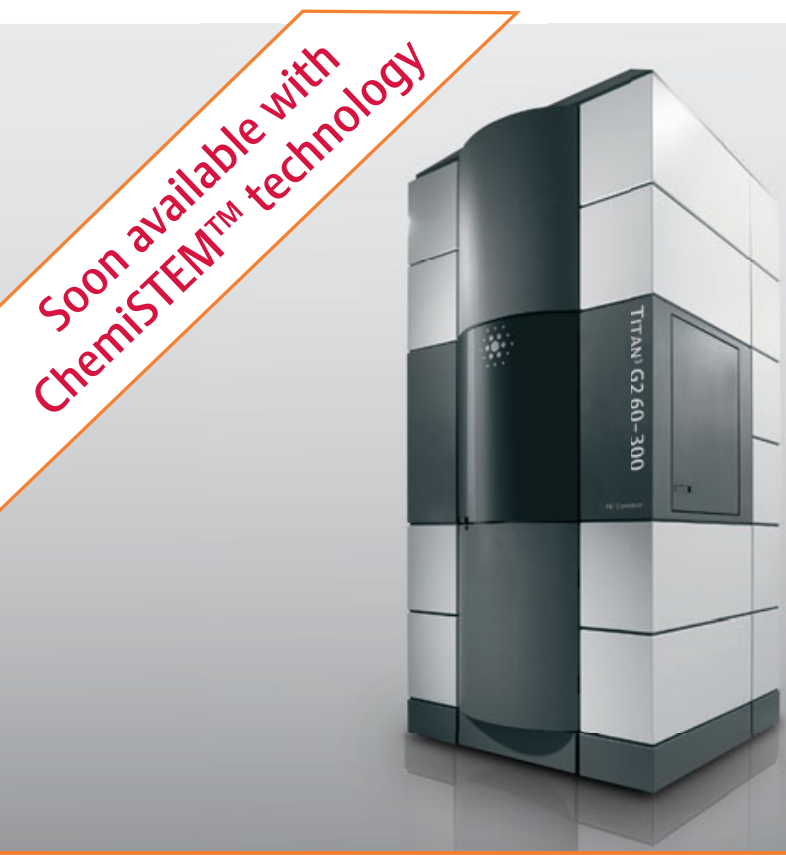

\section{$\operatorname{Titan}^{3^{T M}}$ G2 60-300}

Ultimate performance and high tension flexibility in imaging and analysis in $C_{s}$-corrected S/TEM

- Deep sub-Ångström performance optimized for a wide range of materials

- Monochromator and X-FEG technology for extreme high lateral and energy resolution

- Ultra sensitive ChemiSTEM technology for atomic EDX mapping 